\title{
Scrutiny of the So-Called Einstein's Causality
}

\author{
Mohamed E. Hassani* \\ Institute for Fundamental Research \\ BP.197, CTR, Ghardaïa 47800, Algeria
}

\begin{abstract}
In the present paper, the so-called Einstein's causality is scrutinized and proven to be an illusion. Causality as a well-established universal principle was and is absolutely valid for subluminal, luminal and superluminal signals under any natural and/or artificial circumstances. It is also shown that conceptually special relativity theory (SRT) is inapplicable to superluminality of physical phenomena since SRT has the light speed in vacuum as an upper limiting speed in its own proper domain of applications, and also because SRT is crucially based on the concept of inertial reference frames (IRFs) which are related to each other by Lorentz transformations, that is why the relative velocity of any two IRFs must be smaller than light speed.
\end{abstract}

Keywords: Causality, special relativity theory, light speed in vacuum, superluminal signals

\section{Introduction}

A very old and common misconception about the causality principle and its possible violation by (hypothetical) superluminal signals, which historically goes back to Einstein's thought experiment published in 1907 [1] and translated into English by Schwartz [2]. Afterwards, several distinguished physicists such as Tolman [3], Pauli [4], Von Lau [5], Moller [6], Bohm [7], Penrose [8], and Rindler [9] imitated Einstein without scrutinizing his approach, and created some thought experiments, the so-called (tachyonic) causal paradoxes. Also almost all the mentioned authors as well as the modern ones adopted the same approach that is exclusively based on mathematical fallacy ${ }^{1}$, they are not attached to the context and formalism of SRT. Apparently they are 'deliberately' overlooked: (i) they are dealing with the relative motion in SRT-context, (ii) the physical equivalence of all the IRFs; (iii) universality of relativity principle; (iv) universality of causality principle.

Since then, many textbooks and research articles have completely denied the possibility of signal velocities faster than vacuum speed of light. For example in a paper on 'causality and the Speed of sound' [10], the authors clearly refused to recognize the reality of superluminal physical phenomena because, according to them, causality might be violated if there were really a physical signal faster than $c$. And they refused or omitted to refer to a number of important theoretical and experimental works on the topic published in Nature; Science; Phys. Rev. Lett.; Phys. Lett. A; ApJ ; ... etc., particularly the papers [1133].

The Causality Principle, in the sense of common conventional belief, is an assumption that information traveling faster than light speed in vacuum would represent a violation of causality. However, Causality is simply the idea that the cause of an event precedes the effect of the event. So the common understanding of the causality principle is valid only in the context of special relativity theory (SRT),

\footnotetext{
* hassani641@gmail.com

${ }^{1}$ The traditional way of presenting a mathematical fallacy is to give an invalid step of deduction mixed in with valid steps, so that the meaning of fallacy is here slightly different from the logical fallacy.
} 
wherein Lorentz transformations (LTs) are applicable to inertial reference frames (IRFs) in relative uniform motion at subluminal velocities.

For the causality principle to be really a universal principle, it would have to be equally valid for subluminal, luminal and even superluminal velocities. For instance, suppose a massive particle is emitted before it is absorbed in a detector. Even if the particle's velocity were one trillion times faster than $c$, the cause (emission) would still precede the effect (absorption), and causality would be not violated. For such a case, LTs should be replaced with superluminal spatio-temporal transformations (STs) because, conceptually, the particle in question was moving in superluminal space-time not in Minkowski spacetime. Consequently, in superluminal space-time, "the superluminal signals do not violate the Causality Principle but they can shorten the luminal vacuum time span between cause and effect."

Normally, when we speak about the superluminality of physical phenomena, SRT should not be applied, since it is not concerned with superluminality. SRT is a robust and valid theory only in its own proper domain of applications, i.e., where the relative velocities are relativistic. Hence, superluminal physical phenomena might require study in the framework of another physical theory, which will be structurally built on superluminal space-time as a seat of superluminal events and will have the STs as a cornerstone [11].

It seems that certain authors forgot that any well-established physical theory has its validity limits and its own proper domain of applications. The perpetual development and vivacity of Science is strongly dependent on honoring these validity limits. For example, the validity limits of classical mechanics led to relativistic mechanics. Since the latter has light speed in vacuum as the upper-limit speed thus, as already pointed out, the superluminality does not belong to SRT-domain, and as a result SRT is not related to superluminality of physical phenomena, and conceptually any attempt to apply SRT to superluminal motions would be waste of time.

Einstein himself was clear on this matter because, in order to separate SRT from superluminality, he repeatedly made the following statement: "For velocities greater than that of light our deliberations become meaningless; we shall, however, find in what follows, that the velocity of light in our theory plays the part, physically, of an infinitely great velocity." [34]. Note, however, the occurrence of the expression 'in our theory' this means that the light speed in vacuum is, in fact, seen as an upper limiting speed only in SRT-context, because of its LTs.

The theoretical, observational and experimental evidence of the (apparent) superluminal motions at micro and macroscopic scales allows us to suggest that in Nature there are three kinematical levels (KLs), namely, subluminal-KL, luminal-KL and superluminal-KL in which the physical phenomena may be manifested at subluminal, luminal and superluminal velocities, respectively. Also, each KL should be characterized by its own group of spatio-temporal transformations. For example, subluminal-KL is characterized by the Galilean group for subrelativistic velocities $(v<<c)$ and by Lorentz group for relativistic velocities $(v<c)$, luminal-KL and superluminal-KL would have, respectively, luminal and superluminal groups for luminal $(v=c)$ and superluminal velocities $(v>c)$.

From all that, we arrive, again, at the following result regarding causality: If causality is really a universal principle, it must be valid in all the KLs. Consequently, in such a case, we can say that there are 
in fact three kinds of causality, viz., subluminal causality, luminal causality and superluminal causality, and each kind is characterized by its own proper circumstances.

The main role of each group of spatio-temporal transformations is the study of the chronology of events defined by the couple (cause, effect). This implies that we cannot study, e.g., superluminal causality with the help of Lorentz group, and vice versa, i.e., we cannot apply the group of superluminal transformations to subluminal causality.

\section{Einstein's causality is an illusion}

Returning to causality as a series of events happened in well-defined chronological order. And let us show that Einstein application of the addition theorem of velocities (ATV) to superluminal velocities in order to prove the violation of causality is, indeed, incorrect. Not only because SRT is clearly inapplicable to superluminal motions as we have already seen, but also because superluminal velocities as such should be defined in superluminal space-time not in Minkowski space-time. Thus, conceptually, we cannot apply ATV to superluminal velocities since ATV itself is only valid for subluminal velocities, since the ATV is derived from LTs, which are uniquely valid for subluminal velocities. That is why Einstein [1] had, in this case, rightly affirmed that "A relative motion of reference systems with superluminal velocity is incompatible with our principles."

Therefore, from the above considerations and also as we shall also see, Einstein's proof of causality violation via ATV applied to superluminal velocities is conceptually unphysical.

Let us rewrite Einstein's thought experiment and proof [1] as follows: "From the addition theorem of velocities results the further interesting consequence, that no action can exist which can be utilized for arbitrary signaling and which has a propagation speed greater than that of light in vacuum. In fact, suppose a material strip extended along the $x$-axis of $S$, relative to which a certain action can be propagated with the speed $W$ (as judged from the material strip), and let observers who are at rest relative to $S$ be situated both at the point $x=0$ (point $A$ ) and at the point $x=\lambda$ (point B). Let the observer at $A$ send signals to the observer at $B$ by means of the aforementioned action, through the material strip, which is not at rest but moves with the speed $v(v<c)$ in the direction of the negative $x$ axis. The signal is then, according to the first of equations (3), carried from $A$ to $B$ with the speed $(W-v)\left[1-\left(W v / c^{2}\right)\right]^{-1}$. The time T required for this is therefore $T=\lambda\left[1-\left(W v / c^{2}\right)\right](W-v)^{-1}$.

The speed $v$ can take on any value smaller than $c$. If therefore, as we have assumed, $W>c$, we can always choose $v$ so that $T<0$. This result signifies that we must consider as possible a transmission mechanism that allows the intended action to precede the cause. Although from a purely logical point of view this result does not contain, in my opinion, any contradiction, yet it clashes so much with the character of our whole experience, that the impossibility of the assumption $W>c$ appears thereby to be sufficiently proven."

Einstein wrote the above note in 1907 (see Ref. [1]) -more than a century ago- with the express purpose of showing the clash between causality and superluminal signals. It is clear from the above treatment; Einstein violated his own theory in an unphysical manner, because he deliberately applied the ATV to superluminal velocity to prove the violation of causality by superluminal signals. However, this 
way in which the proof was done constitutes a major contradiction from the physical viewpoint, particularly, when we take into account the fact that the light speed in vacuum is an upper limiting speed in SRT. Furthermore, as it was said, superluminal signals do not propagate in Minkowski space-time, but in superluminal space-time, as the seat in which superluminal physical phenomena may occur.

\section{Mathematical viewpoint}

If we put Einstein's treatment under closer scrutiny especially the expression "If therefore, as we have assumed, $W>c$, we can always choose $v$ so that $T<0$ ", we find that, for Einstein, the inequality $T<0$ is a criterion, or sufficient condition, to say: the effect precedes the cause, and consequently causality is violated by superluminal velocity.

\subsection{First counterexample}

However, mathematically, we can always get the same inequality $T<0$ even for subluminal signal velocity, i.e., when $W<c$. To this end, let $\forall k, k^{\prime} \in(1,+\infty) \subset \mathbf{R}: k>k^{\prime}$ so that $W=c / k$ and $v=c / k^{\prime}$. Thus after substitution we get

$$
T=\left(1-\frac{1}{k k^{\prime}}\right)\left(\frac{1}{k}-\frac{1}{k^{\prime}}\right)^{-1} \frac{\lambda}{c}<0 .
$$

Since there exist an infinite number of couple $\left(k, k^{\prime}\right)$ that satisfies the above conditions, we can affirm that, by assuming $W<c$, we can always choose $v$ to have $T<0$. Therefore, this counterexample shows us that the inequality $T<0$ cannot hold the status of a criterion or sufficient condition to prove the violation of causality.

\subsection{Second counterexample}

This second counterexample is judged more important because mathematically, and only mathematically would demonstrate that even for superluminal signal velocity, i.e., when $W>c$, we can always choose $v$ so that $T>0$. With this aim, let $\forall k, k^{\prime} \in(1,+\infty) \subset \mathbf{R}: k<k^{\prime}$ such that $W=k c$ and $v=c / k^{\prime}$. Hence following substitution, we find

$$
T=\left(1-\frac{k}{k^{\prime}}\right)\left(k-\frac{1}{k^{\prime}}\right)^{-1} \frac{\lambda}{c}>0 .
$$

In view of the fact that there is an infinite number of couple $\left(k, k^{\prime}\right)$ fulfills the above conditions, therefore, we can mathematically state that by supposing $W>c$, we can always choose $v$ to have $T>0$.

Finally, as a pedagogical illustration, we have listed in Tables 1 and 2 - according to the conditions of the first and second counterexamples - some numerical values for the inequalities (1) and (2). 
5

\begin{tabular}{ccc}
\hline \hline$k$ & $k^{\prime}$ & $T$ \\
& & $(\lambda / c)$ \\
\hline 1.50 & 1.25 & -03.50 \\
1.75 & 1.50 & -06.50 \\
2.00 & 1.75 & -10.00 \\
2.25 & 2.00 & -14.00 \\
2.50 & 2.25 & -18.00 \\
2.75 & 2.50 & -23.50 \\
3.00 & 2.75 & -29.50 \\
3.25 & 3.00 & -35.00 \\
3.50 & 3.25 & -41.51 \\
3.75 & 3.50 & -48.51 \\
& & \\
\hline \hline
\end{tabular}

Table 1: Some numerical values for the inequality (1) when the signal is supposed subluminal.

\begin{tabular}{ccc}
\hline \hline$k$ & $k^{\prime}$ & $T$ \\
& & $(\lambda / c)$ \\
\hline 1.50 & 2.00 & \\
2.00 & 2.50 & $2.500 \times 10^{-1}$ \\
2.50 & 3.00 & $1.250 \times 10^{-1}$ \\
3.00 & 3.50 & $7.694 \times 10^{-2}$ \\
3.50 & 4.00 & $5.263 \times 10^{-2}$ \\
4.00 & 4.50 & $3.846 \times 10^{-2}$ \\
4.50 & 5.00 & $2.941 \times 10^{-2}$ \\
5.00 & 5.50 & $2.325 \times 10^{-2}$ \\
5.50 & 6.00 & $1.886 \times 10^{-2}$ \\
6.00 & 6.50 & $1.562 \times 10^{-2}$ \\
& & $1.315 \times 10^{-2}$ \\
\hline \hline
\end{tabular}

Table 2: Some numerical values for the inequality (2) when the signal is supposed superluminal. 


\section{Physical viewpoint}

In spite of the fact that the superluminality of physical phenomena is beyond the scope of SRT, nevertheless, if we put the concept of proper (inertial reference) frame or comoving ${ }^{2}$ (IR) frame aside, we can disprove Einstein's claim in the context of SRT. To this end, we should take into account the physical equivalence of all the IRFs, universality of relativity principle, universality of causality principle, and the following LTs in their usual form:

$$
S \rightarrow S^{\prime}:\left\{\begin{array}{l}
x^{\prime}=\gamma(x-v t) \\
y^{\prime}=y \\
z^{\prime}=z \\
t^{\prime}=\gamma\left(t-\frac{v x}{c^{2}}\right)
\end{array} \quad S^{\prime} \rightarrow S:\left\{\begin{array}{l}
x=\gamma\left(x^{\prime}+v t^{\prime}\right) \\
y=y^{\prime} \\
z=z^{\prime} \\
t=\gamma\left(t^{\prime}+\frac{v x^{\prime}}{c^{2}}\right)
\end{array}\right.\right.
$$

where $\gamma=1 / \sqrt{1-\beta^{2}}, \beta=v / c,-c<v<c$, and the two IRFs $S$ and $S^{\prime}$ are supposed in standard configuration. Besides, it is well-known that LTs have an algebraic structure of orthogonal-orthochronous group. This means, among many other interesting properties, LTs preserve the direction of time or equivalently the notions of 'past' and 'future' for time intervals are perfectly preserved. That is:

$$
\begin{aligned}
& \text { if } \Delta t^{\prime} \leq 0 \text { in } S^{\prime} \Rightarrow \Delta t \leq 0 \text { in } S, \\
& \text { if } \Delta t^{\prime} \geq 0 \text { in } S^{\prime} \Rightarrow \Delta t \geq 0 \text { in } S,
\end{aligned}
$$

and vice versa, that is

$$
\begin{aligned}
& \text { if } \Delta t \leq 0 \text { in } S \Rightarrow \Delta t^{\prime} \leq 0 \text { in } S^{\prime}, \\
& \text { if } \Delta t \geq 0 \text { in } S \Rightarrow \Delta t^{\prime} \geq 0 \text { in } S^{\prime} .
\end{aligned}
$$

Furthermore, since the universality of causality principle is intimately related to the universality of relativity principle which together imply the following condition: If a hypothetical subluminal or superluminal signal propagates at velocity $u<c$ or $u>c$ relative to $S$, also it should propagate at subluminal or superluminal velocity $u^{\prime}<c$ or $u^{\prime}>c$ relative to $S^{\prime}$. Actually, this 'causality-relativity' condition is a direct consequence of LTs. More precisely, the orthogonality property of LTs preserve the invariance of Minkowski differential quadratic form in all the IRFs:

$$
d x^{\prime 2}+d y^{\prime 2}+d z^{\prime 2}-c^{2} d t^{\prime 2}=d x^{2}+d y^{2}+d z^{2}-c^{2} d t^{2},
$$

which can be written into the form

$$
\left(u^{\prime 2}-c^{2}\right) d t^{\prime 2}=\left(u^{2}-c^{2}\right) d t^{2}
$$

\footnotetext{
${ }^{2}$ A proper (IR) frame, or comoving (IR) frame, is a frame of inertial reference that is attached to an object. The object in this frame is stationary within the frame, which is useful for many types of calculations. Consequently, you are certainly aware that $\gamma=\left(1-v^{2} / c^{2}\right)^{-1 / 2}$, the ratio between coordinate and proper time $(d t / d \tau)$, diverges as $v \rightarrow c$. The inequality $v>c$ leads to a purely imaginary $\gamma$, therefore, the relative velocity of two IRFs must be smaller than $c$. Consequences: since an IRF can be associated with any non-accelerated particle or material object moving with subluminal velocity, i.e., $|v|<c$, this statement -in the context of SRT- translates into the requirement that the velocity of material objects and of all physical signals be limited by $c$.
} 
Once again, at this point we should notice the important fact that subluminal velocities always transform to subluminal velocities and superluminal velocities to superluminal velocities. For if $u$ and $u^{\prime}$ are velocities of hypothetical particle or signal in $S$ and $S^{\prime}$, respectively.

Let us return to Einstein's thought experiment and proof where the moving material strip plays the role of IRF $S^{\prime}$ which is in relative motion at velocity $v(v<c)$ in the direction of the negative $x$-axis. Therefore, if we take into account the universality of causality principle and relativity principle, we find that if the hypothetical superluminal signal propagates at velocity $W>c$ relative to $S$ (stationary material strip), thus also it should propagate at superluminal velocity $(W-v)\left[1-\left(W v / c^{2}\right)\right]^{-1}>c$ relative to $S^{\prime}$ (moving material strip). Now, let us focus our interest on the above inequality which has completely escaped Einstein's attention, and notice that since $W>c, v<c$ and $(W-v)\left[1-\left(W v / c^{2}\right)\right]^{-1}>c$ this obviously implies that both numerator and denominator in the inequality are positive, i.e., $W-v>0$ and $1-\left(W v / c^{2}\right)>0$ from where we get the following important conditions: $W v<c^{2}$ and $v<\frac{c^{2}}{W}$. This means all the possible values of $v$ belong to the interval $\left[0, \frac{c^{2}}{W}\right)$. Consequently, $\forall v \in\left[0, \frac{c^{2}}{W}\right)$ we get always

$$
T=\lambda\left[1-\left(W v / c^{2}\right)\right](W-v)^{-1}>0 .
$$

That is quite contrary to Einstein's claim [The speed $v$ can take on any value smaller than $c$. If therefore, as we have assumed, $W>c$, we can always choose $v$ so that $T<0$.] because $v$ cannot take any value (smaller than $c$ ) outside the interval $\left[0, \frac{c^{2}}{W}\right.$ ).

\section{Discussion}

Between the lines of Einstein's proof there is a contradiction. Firstly, it seems that Einstein was not seriously attached to the context and formalism of his thought experiment because his claim [ ... If therefore, as we have assumed, $W>c$, we can always choose $v$ so that $T<0$.$] is clearly inconsistent$ with the fact that, according to his thought experiment, the hypothetical signal is carried from $A$ to $B$ at superluminal velocity $(W-v)\left[1-\left(W v / c^{2}\right)\right]^{-1}>c$ relative to the moving material strip which plays the role of IRF $S^{\prime}$. Secondly, The expression itself of the above inequality has a mathematical and physical sense only if $0 \leq v<\frac{c^{2}}{W}$. This indicates that the interval of all the possible values of $v$ is a priori and implicitly contained in that inequality. Consequently, $v$ cannot take any value (smaller than $c$ ) outside the interval $\left[0, \frac{c^{2}}{W}\right)$. However, we can always choose $v$ from the interval $\left[0, \frac{c^{2}}{W}\right)$ to have $T>0$.

From all that, we can affirm that the superluminal influences in general and superluminal signals in particular are in perfect harmony with relativity principle and causality principle, since the concept of infinite velocities is a purely mathematical one. Moreover, the authors who repetitively claimed that the superluminal influences of any sort would be inconsistent with SRT, their objections rest upon very 
elementary common misunderstanding of Minkowski geometry, LTs, and relativistic kinematics. Also they 'deliberately' overlooked the equivalence of all the IRFs, universality of relativity principle, universality of causality principle, and the fact that any well-established physical theory has its validity limits and its own proper domain of applications.

\section{Conclusion}

This analysis has challenged the denial of the physical reality of superluminality at micro and macroscopic levels, and the refusal to recognize the real possibility of superluminal signals that appears in many textbooks and research articles. The so-called Einstein's causality, and its supposed violation, are both pure illusions - a sort of mathematical fallacy. It has also shown that the theoretical, observational, and experimental evidence of superluminal motions did not threaten SRT, because the superluminality of physical phenomena did not belong to the SRT-domain of applications, since SRT has the light speed in vacuum as an upper limiting speed; therefore, any attempt to apply SRT to superluminality will be a complete waste of time because, once again, as Einstein [1] had, in this sense, correctly affirmed that " $A$ relative motion of reference systems with superluminal velocity is incompatible with our principles." since SRT is crucially based on the concept of IRFs. Finally, we need to formulate an original and radical theory capable of studying, explaining, and predicting the superluminal physical phenomena [11]. Of course, as it was said, the expected physical theory should be fundamentally based on superluminal spacetime as the seat of superluminal events and should have the superluminal spatio-temporal transformations as a cornerstone.

\section{References}

[1] A. Einstein, Jaharb. Radioakt. Elektron. 4, 411 (1907)

[2] H. M. Schwartz, Am. J. Phys., 45, 512 (1977)

[3] R. C. Tolman, The Theory of relativity of Motion, Berkeley (1917)

[4] W. Pauli, Relativitätstheorie, Leipzig (1921)

[5] M. von Laue, La Théorie de la Relativité, Gauthier-Villars, Paris (1922)

[6] C. Møller, The theory of relativity, Clarendon Press, Oxford (1952)

[7] D. Bohm, The Special Theory of Relativity, Routledge (1996) (first edition, W. A. Benjamin, Inc. (1965))

[8] R. Penrose, The Emperor's New Mind, University Press, Oxford (1989)

[9] W. Rindler, Relativity - Special, General, and Cosmological 2nd ed. University Press, Oxford (2006)

[10] G.F.R Ellis, R. Maartens and A.H. Malcolm, arXiv: 0703121v2 (2007)

[11] M. E. Hassani, Comm. in Phys., 24, 313 (2014)

[12] G. Nimtz and A. Enders, J. Phys. I (France) 2, 1693 (1992)

[13] A. Ender and G. Nimtz, Phys. Rev. B, 47, 9605 (1993)

[14] A. Ender and G. Nimtz, J. Phys. I (France) 3, 1089 (1993) 
[15] G. Nimtz, A. Enders and H. Spieker, J. Phys. I (France) 4, 565 (1994)

[16] G. Nimtz and A. Haibel, arXiv:physics/0104063v1 (2001)

[17] G. Nimtz and W. Heitmann, Prog. Quantum Electron. 21, 81 (1997)

[18] L. J. Wang, A. Kuzmich and A. Dogariu, Nature, 406, 277 (2000)

[19] T. J. Pearson et al, Nature, 290, 365 (1981)

[20] A. Brunthaler et al, A\&A 357, L 45 (2000)

[21] I. F. Mirabel and L. F. Rodriguez, Nature 371, 46 (1994)

[22] S. J. Newell, M. A. Garrett and R. E. Spencer, MNRAS 293, L17 (1998)

[23] U.S. Inan et al, Geophys. Res. Lett. 23, 133 (1996)

[24] F. A. Jenet, et al., ApJ 710, 1718 (2010)

[25] C.C. Cheung et al., ApJ 663, L65, (2007)

[26] H. Ardavan et al., MNRAS 388, 873, (2008).

[27] H. Ardavan et al., J. Opt. Soc. Am. A 24, 2443 (2007)

[28] H. Ardavan et al., J. Opt. Soc. Am. A 25, 780 (2008)

[29] H. Ardavan et al., Journal of Mathematical Physics 50, 103510 (2009)

[30] A. Ardavan et al., J. Appl. Phys. 69(12) 7760, (2004)

[31] H. Ardavan, Phys. Rev. E 58(5), 6659 (1998)

[32] A. Ranfagni et al., Phys. Rev. E 48, 1453 (1993)

[33] A. Haché and L. Poirier, Appl. Phys. Lett. 80, 518 (2002))

[34] A. Einstein, Ann. Phys. (Leipzig) 17, 891 (1905) 